\title{
Evaluation of stem-like side population cells in a recurrent nasopharyngeal carcinoma cell line
}

\author{
Susan Ling Ling Hoe ${ }^{1,2}$, Lu Ping Tann ${ }^{1}$, Juliana Jamal ${ }^{1,5}$, Suat Cheng Peh ${ }^{3}$, Ching Ching Ng${ }^{2}$, Wen Cai Zhang ${ }^{4}$, \\ Munirah Ahmad ${ }^{1}$ and Alan Soo Beng Khoo ${ }^{1 *}$
}

\begin{abstract}
Background: Side population (SP) assay identifies cells with dye/drug extrusion ability, a characteristic of stem cells. Here, we determined if SP cells exist in a verified cell line originating from recurrent nasopharyngeal carcinoma (NPC) and a xenograft established from recurrent metastatic NPC. These cells were evaluated for stem-like properties via functional assays as well as for tumourigenicity.

Methods: We used Hoechst 33342 to identify the SP from non-SP (NSP) cells in HK1 NPC cell line and xeno-284 NPC xenograft. The cells were assayed for in vitro characteristics of cancer stem cells (CSC), gene expression and tumourigenicity ability. Student's test was used to test for significance.

Results: Five to ten percent and less than $0.5 \%$ of HK1 and xeno-284 NPC cells, respectively, were SP cells. Fumitremorgin C (FTC), as opposed to verapamil, was effective in causing the cells to retain Hoechst 33342 dye. HK1 SP cells formed more holoclones, had more aldehyde dehydrogenase (ALDH) activity, divided asymmetrically and contained slow-proliferating cells. ABCG2, SOX2, TERT, MYC, Hedgehog, Notch, TGF $\beta$ and Wht signalling pathway genes were significantly upregulated in the SP cells. However, despite these differences in vitro, both HK1 SP and NSP cells had an overall similar tumourigenic potential in vivo.

Conclusions: HK1 SP cells were ABCG2-specific as confirmed by FTC inhibition and gene expression data. Despite data from in vitro and gene expression experiments suggesting stem-like features, there was no significant difference in tumourigenic potential between SP and NSP cells. We conclude that SP assay alone is not sufficient to identify CSCs in HK1 cells. Our work also suggests the presence of a stem-cell like population among NPC cells which do not display increased tumourigenicity.
\end{abstract}

Keywords: Side population, Nasopharyngeal carcinoma, Slow-cycling, Stem-like, Cancer stem cells

\section{Background}

Nasopharyngeal carcinoma (NPC) is the most common malignancy arising from the nasopharynx and its causation is closely associated with the Epstein-Barr virus, environmental as well as dietary factors [1]. Majority of NPC cases present in late stages [2]. The late presentation of the disease is due to the hidden location of the tumour, which could present with either no or apparently trivial symptoms which could be dismissed by patients or even medical professionals [3]. In addition, disease recurrence,

\footnotetext{
* Correspondence: alankhoo@imr.gov.my

1 Molecular Pathology Unit, Cancer Research Centre, Institute for Medical Research, Jalan Pahang, 50588 Kuala Lumpur, Malaysia

Full list of author information is available at the end of the article
}

therapeutic resistance and metastasis remain major clinical problems [4].

The cancer stem cell (CSC) model hypothesizes that there is a hierarchy within the tumour cell population and only a rare subset of cancer cells has the ability to self-renew and to differentiate, leading to the recapitulation of the original tumour [5]. As such, the spread of CSCs is an important component of the process of metastasis and, reactivation of CSC proliferation is believed to be the underlying cause of disease recurrence.

CSCs are found to behave differently from the rest of tumour cells; amongst others, they have enriched tumourforming potential, and have efficient drug extrusion systems to evade most chemotherapeutic drugs [6]. These cells undergo asymmetric divisions to give rise to daughter 
cells: one which will be stem-like and, the other which does not show stem cell characteristics. Some CSCenriched subpopulations were slower in proliferation (reviewed in Moore et al. [7]), whilst others reported an equal or rapid proliferation rate than the non-CSC subpopulations $[8,9]$.

As drug extrusion is one of the properties for CSC, several groups had adapted the original side population (SP) assay by Goodell et al. [10] for identification of putative stem cells and progenitors in solid tumours [11-13]. The ability of SP cells to extrude the Hoechst 33342 dye, causing them to appear as a dimly stained side population in flow cytometry dot plots, is dependent on the activity of the ATP-binding cassette $(\mathrm{ABC})$ transporter family which includes ABCB1, ABCC1 and ABCG2 [14]. Verapamil is a potent inhibitor for $\mathrm{ABCB} 1$ which also weakly inhibits ABCG2 activities, while fumitremorgin C (FTC) specifically inhibits ABCG2 [15,16]. By adding one of these inhibitors into the SP assay, one can determine the type of $\mathrm{ABC}$ transporter protein which is responsible for the dye extrusion activity. Prior to this report, there was an earlier publication on the use of SP assay in well- and poorlydifferentiated NPC cell lines which indicated that putative $\mathrm{CSC}$ in these cell lines may be related to ABCB1 activities [17]. However, in vivo tumourigenicity assay was performed for only 4 weeks and the identity of the cell line chosen to perform downstream functional experiments was questioned in a later publication [18].

NPC HK1 is a cell line established from a welldifferentiated recurrent NPC sample [19], while xeno284 is a xenograft line established in our laboratory from a poorly differentiated recurrent metastatic NPC sample. In this study, we first tested for the presence of the SP subpopulation in HK1 and xeno-284 NPC cells, followed by sorting of SP and non-SP (NSP) subpopulations for comparison of in vitro clone morphology, cell division and proliferation. Aldehyde dehydrogenase (ALDH) flowstaining was carried out to determine the level of ALDH activity in the sorted cells. Gene expression studies were also performed to identify stem cell related genes and pathways which may be responsible for the observations. Finally, in vivo tumourigenicity experiments were performed for duration of up to 7 weeks to evaluate the tumourinitiating ability of SP and NSP cells.

\section{Results}

\section{HK1 contains SP cell subpopulation}

The identity of the HK1 NPC cells used was validated by short tandem repeat (STR) profiling to be identical to the HK1 cells used by others [18] (Additional file 1). The SP phenotype as identified by low Hoechst 33342 blue/red fluorescence intensity was detected in $5-10 \%$ of HK1 cells (Figure 1). The loss of the SP population with addition of FTC but not verapamil, suggested that ABCG2 was the functional ABC transporter in these SP cells (Figure 1). Compared to HK1, xeno-284 cells had very few (less than $0.5 \%$ ) or no SP cells during replicate runs (Figure 1). As such, only HK1 SP and NSP cells were used for subsequent downstream experiments.

\section{HK1 SP cells form holoclones during in vitro culture}

Sorted SP and NSP cells exhibited different growth patterns. After a week of in vitro culture in fully-supplemented RPMI medium, most of the SP cells grew into holoclones which formed tightly-clustered cells with well-defined clone borders (Figure 2A). Clones established by the NSP cells primarily consisted of slightly-scattered cells with irregular borders (meroclones) and/or tiny clusters of cells which did not display much growth (paraclones) (Figure 2B). Repeated experiments showed that SP cells formed more holoclones than NSP cells ( $\mathrm{p}<0.0001$; Figure $2 \mathrm{C}$ ).

\section{HK1 SP cells express higher aldehyde dehydrogenase activity}

The ALDEFLUOR staining kit used in this study detects the enzymatic activity of ALDH1, a marker of stemness [20]. Cells incubated with the ALDEFLUOR substrate (BAAA) and the ALDH inhibitor (DEAB) were used to define the ALDH ${ }^{\text {bright }}$ region (representative plots as shown in Figure 2D). The SP cells showed a significantly higher population of ALDH ${ }^{\text {bright }}$ cells $(18.08 \pm 11.46 \%)$ than NSP cells $(5.10 \pm 3.56 \%)$ (Figure $2 \mathrm{E})$.

\section{HK1 SP cells divide asymmetrically into SP and NSP cells during in vitro culture}

The ability of SP and NSP cells to grow and divide asymmetrically in vitro was examined by re-evaluating the percentages of SP and NSP fractions in the sorted cells. After three weeks of culture, the SP sorted cells had divided into both SP and NSP cells, with only $24.57 \pm 7.97 \%$ of the cells still remaining as SP cells. On the other hand, $6.07 \pm 1.74 \%$ of SP cells had reappeared in the NSP sorted cells (Figure 3A).

\section{HK1 SP cells proliferate slower than NSP cells}

There was a significant difference in the normalized growth rates between SP and NSP cells in the impedance-based assay $(\mathrm{p}<0.0001$; Figure $3 \mathrm{~B})$. SP cells grew at a slower rate as compared to NSP cells especially at the first 72 hours of observation. At the end of the experiment, growth rates of the SP cells and NSP cells were getting similar to the growth rate of control cells.

Analysis of stem cell related genes in HK1 SP and NSP cells A total of 168 genes related to stem cell identification and/or involved in key stem cell pathways were analysed by quantitative RT-PCR. Fifty genes were significantly upregulated by at least 2 fold in SP cells compared to 


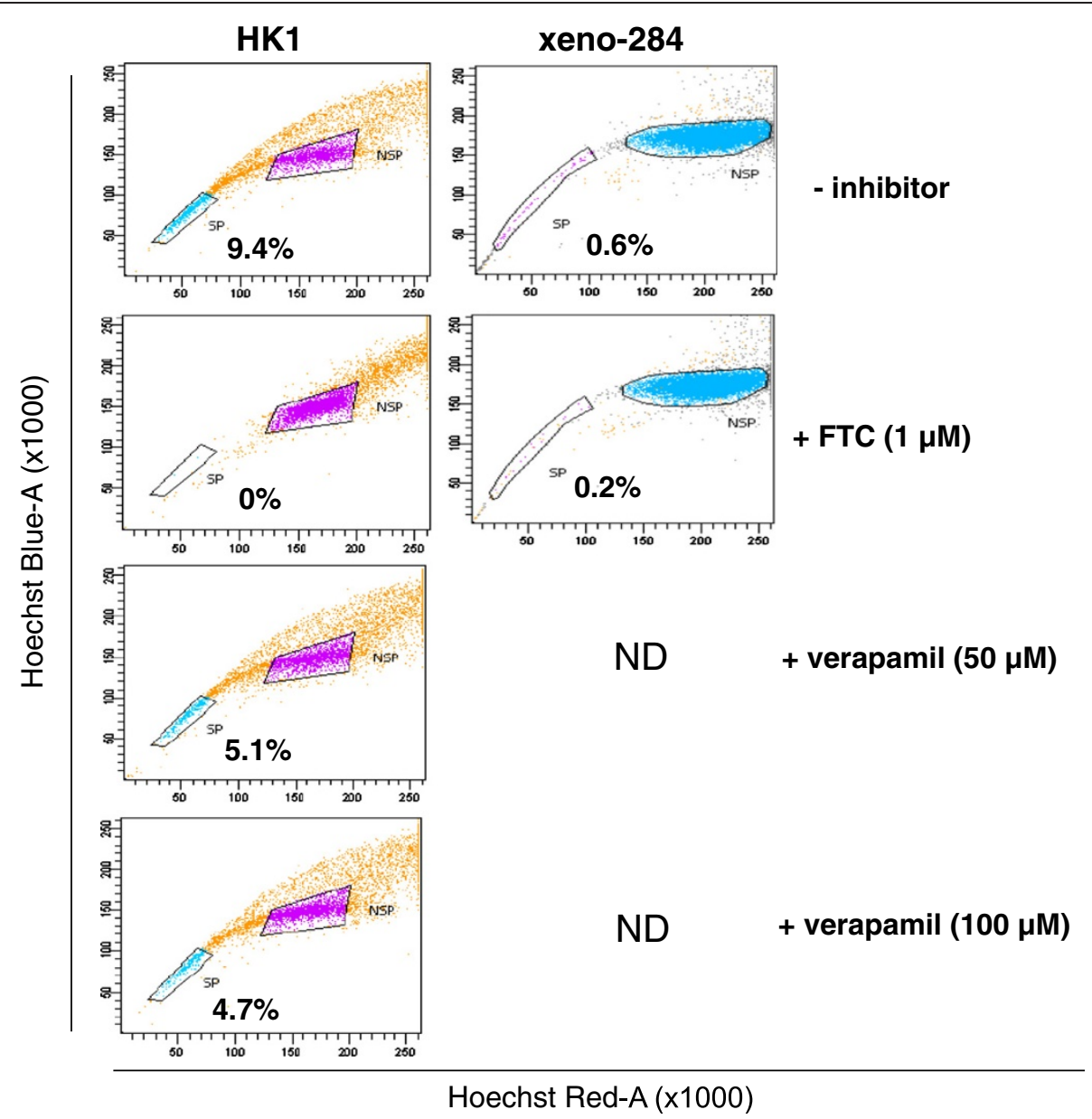

Figure 1 Identification of side population in NPC cells. Representative dot plots of HK1 and xeno-284 NPC cells stained with Hoechst 33342 dye, with and without inhibitor. The inhibitory effect of FTC at $1 \mu \mathrm{M}$ was more evident in HK1 cells as compared to verapamil at both concentrations of 50 and $100 \mu \mathrm{M}$. ND: not done.

NSP cells; amongst them were $A B C G 2, S O X 2, M Y C, T E R T$ and, members of the Hedgehog (GLI1-3, PTCH1, PTCHD2 and $S U F U)$, Notch, TGF $\beta$ ( $A C V R 1 B, S M A D 1, S M A D 7$ and LTBP2-3) and Wnt (FZD2, FZD6-7, BCL9 and BCL9L) signalling pathways (Figure 4; Additional file 2). None of the genes tested were significantly downregulated in SP cells.

HK1 SP subpopulation does not display significant enrichment for tumour-initiating cells in an in vivo xenograft assay

As the in vitro experiments suggested that the SP subpopulation enriched for cells with stem-like phenotype, we proceeded to determine whether this subpopulation would enrich for tumour-initiating cells in an in vivo assay.

A pilot study showed that 1000 unsorted HK1 cells could form tumours in nude mice (data not shown). To determine the tumour-forming ability of SP and NSP cells, 1000,100 and 10 cells were inoculated subcutaneously into nude mice (Figure 5A). Three out of four tumours from inoculation of 10 SP cells which were detected earlier than the first tumour from inoculation of NSP cells of the same number suggested that SP cells may have a growth advantage compared to NSP cells. The growth advantage was however lost at higher inoculations of 100 and 1000 cells. Limiting dilution analysis using the Extreme Limiting Dilution Analysis (ELDA) software showed that the estimated number of tumour-initiating cells based on the in vivo assay was not significantly different between the SP and NSP subpopulations, implying that the difference in the potential of SP and NSP cells to form tumours was not significant (Figure 5B). Both SP and NSP tumours showed similar histomorphology as seen in unsorted HK1 cells grown in mice (Figure $5 \mathrm{C}$ ). Histologically, there was also no substantial difference between SP and NSP tumours in the degree of differentiation, stromal reaction and cell pleomorphism (Figure 5D), except for one SP tumour which showed vascular invasion (Figure 5 Div). 

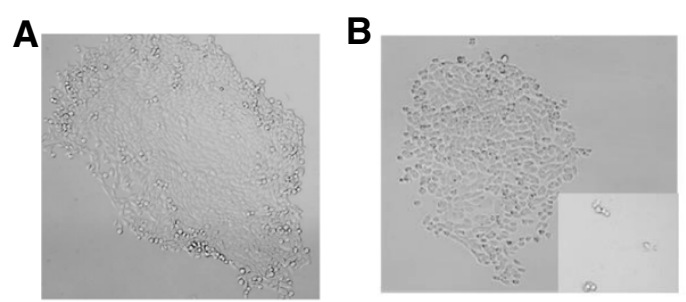

\section{D}

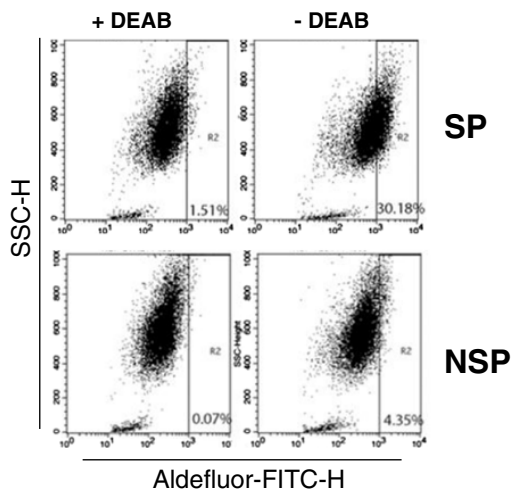

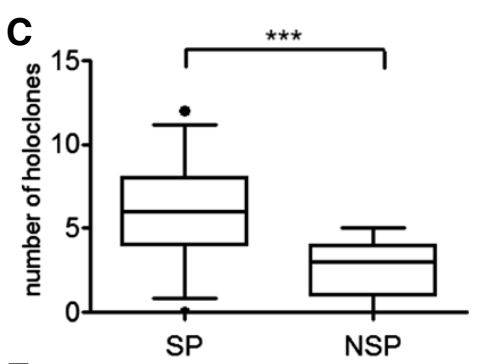

E

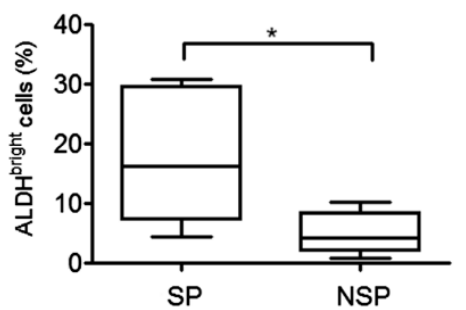

Figure 2 SP subpopulation enriches for stem-cell like phenotype in in vitro assays. (A) During in vitro culture, majority of SP cells formed holoclones with individual cells clustering tightly and forming a well-defined clone border. (B) NSP cells tended to form loose clusters of meroclones or, paraclones (inset) with tiny clusters of cells situated far from each other. All photos were taken on day 9 post-sorting; original magnification 100X. (C) SP cells formed more holoclones than NSP cells during in vitro culture $(p<0.0001)$. (D) The gating for the ALDHbright population was set by a control tube with the addition of DEAB, an ALDH inhibitor. (E) SP cells showed higher presence of ALDH ${ }^{\text {bright }}$ cells $(18.08 \pm 11.46 \%)$ than NSP cells $(5.10 \pm 3.56 \%)(p<0.05)$.

\section{Discussion}

CSCs are frequently identified by the presence of surface antigens/markers associated with self-renewal, differentiation, proliferation, metastasis and/or drug resistance characteristics, having increased ALDH activity or through their ability to extrude drugs by $\mathrm{ABC}$ transporters. However, these approaches may not be universal in identifying the same phenotype (s) of CSCs within a particular cancer (reviewed in [21]). In NPC, different CSC markers had been reported from the same cell lines generated from primary NPC cases (Additional file 3) [17,22-25]. In this study, we aim to evaluate the presence of CSCs in a cell line established from recurrent NPC by the SP approach.

Results from our various in vitro-based assays indicate that the SP cells of HK1 were stem-like. During a week's growth in fully-supplemented RPMI medium, both SP and
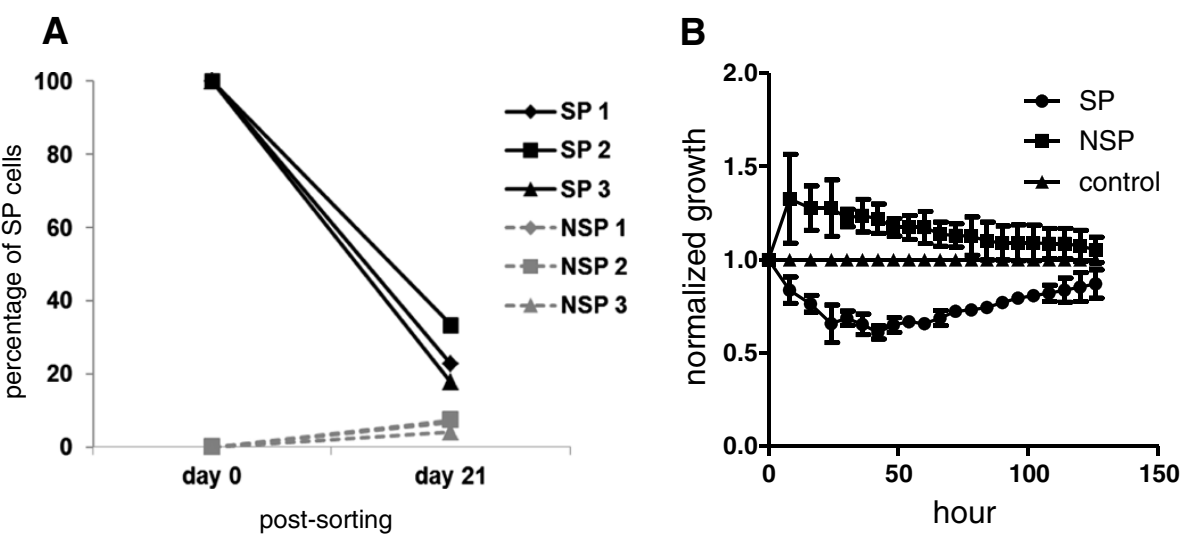

Figure 3 SP cells grow at a slower rate compared to NSP cells. (A) Upon relabelling with Hoechst 33342 on day 21 post-sorting, recultured SP cells had divided asymmetrically into SP and NSP phenotypes. Recultured NSP cells had poorer asymmetric division ability. (B) Impedance-based cell growth assay indicated that the normalized growth rate for SP cells was lower than NSP cells. 


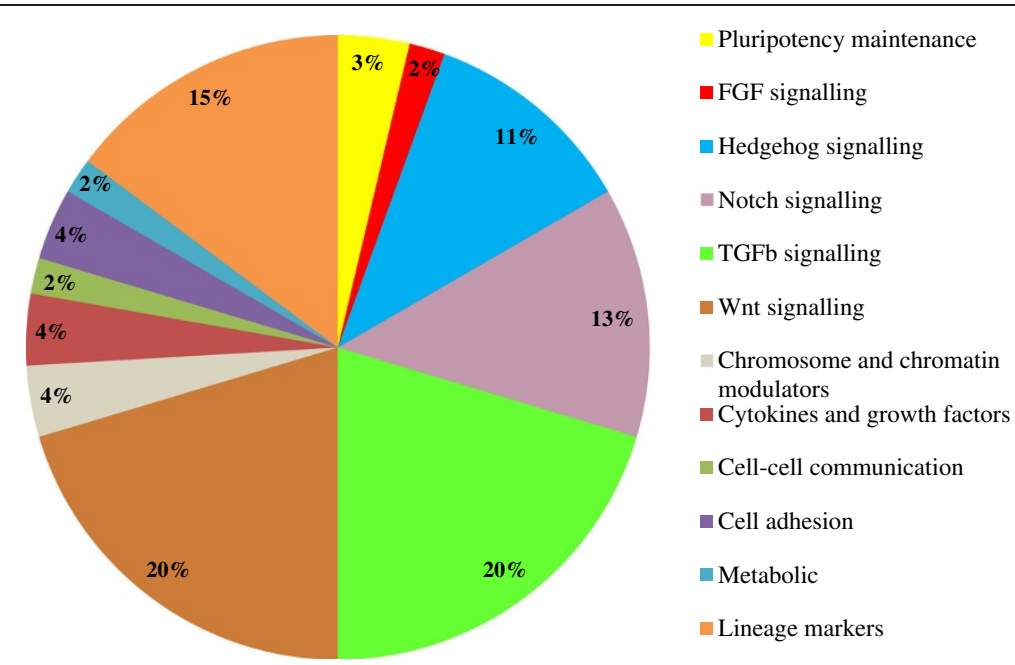

Figure 4 SP subpopulation enriches for cells expressing stem-cell related genes. Combined data from Stem Cell PCR Array and Stem Cell Signaling PCR Array were clustered according to gene function or signaling pathways. Gene expression data were normalized to five housekeeping genes via geometric mean calculation. Only genes which were significantly upregulated by at least 2 fold, with raw Ct values of less than 35 are shown here. None of the downregulated genes fulfilled the selection criteria of at least 2 fold change and $p<0.05$. Some of the genes appeared in more than 1 gene function or signaling pathway (refer to Additional file 2 for details).

NSP cells exhibited different morphology of the colonies in in vitro culture. SP cells grew into tightly packed clusters of holoclones, a particular clonal growth morphology closely associated with stem cells and self-renewal ability, as opposed to NSP cells which mostly grew as meroclones and/or paraclones [26-28]. SP cells in HK1 were also found to be significantly enhanced with ALDH activity, which is known to enhance cancer cell survival and therapy resistance [20]. Gene expression analysis showed upregulation of $A B C G 2$ mRNA level in HK1 SP cells which further indicated the type of $\mathrm{ABC}$ transporter being studied here. These SP cells also showed increased mRNA levels for SOX2, MYC and TERT which were associated with CSC biology and maintenance of pluripotency state [29,30]. Over-expression of Hedgehog pathway genes such as GLI1, GLI2, GLI3FL, PTCH1 and PTCHD2 was in line with reports that Hedgehog pathway can induce $A B C G 2$ expression [31] and it was activated in stem-like cells in EBV positive NPC [23,32].

Nonetheless, there were other findings in our study which seemingly suggested that the stem-like characteristic of SP cells in HK1 may be transient. SP cells of HK1 grew slower in culture which is in line with reports that CSCs from various tumours had slower proliferation rate and hence evaded chemotherapeutic agents which targeted fast growing cells [33,34]. However, the slower rate gradually diminished after a week (Figure 3B). Also, in long term culture, NSP cells gave rise to SP cells (Figure 3A). Together, these two time course in vitro experiments (proliferation assay and asymmetry assay) indicated that the difference between SP and NSP sorted cells may be transient, as both type of cells could interconvert $[17,35]$.

The indication that SP cells can only maintain a transient stem-like phenotype in HK1 might well explain the discordance of our in vitro results with in vivo results. Although there was a growth advantage of SP cells in the lowest inoculation group of mice, an overall comparable tumourigenic potential of both SP and NSP cells was recorded after 7 weeks of in vivo transplantation (Figure 5). The discordance of in vitro and in vivo results is not uncommon, as it has been reported in studies utilizing SP approach to identify CSC in thyroid cancer [36] and cervical cancer [37] as well as studies using marker approach to identify CSC in NPC [25] and colorectal cancer [38]. In our study, although HK1 SP cells were significantly enriched for ALDH ${ }^{\text {bright }}$ cells, there were $5 \%$ of ALDH ${ }^{\text {bright }}$ cells in NSP cells. Wu et al. had reported that ALDH positive cells are CSCs in NPC [22]. If ALDH positive cells were also CSCs in recurrent NPC, this would lend credence to our observation of HK1 NSP cells' ability to generate tumour growth may be partially due to the subset of ALDH positive cells in them.

Our negative findings in in vivo study suggested that SP approach in HK1 cells only enriches for a certain subtype with stem-cell features but not bona fide CSCs as depicted by both SP and NSP subpopulations' ability to confer tumour growth in mice. This is in contrast to an earlier report that showed significant tumour growth difference of SP cells over NSP cells in NPC [17]. The disparity may be related to firstly, the type of NPC cell line being 


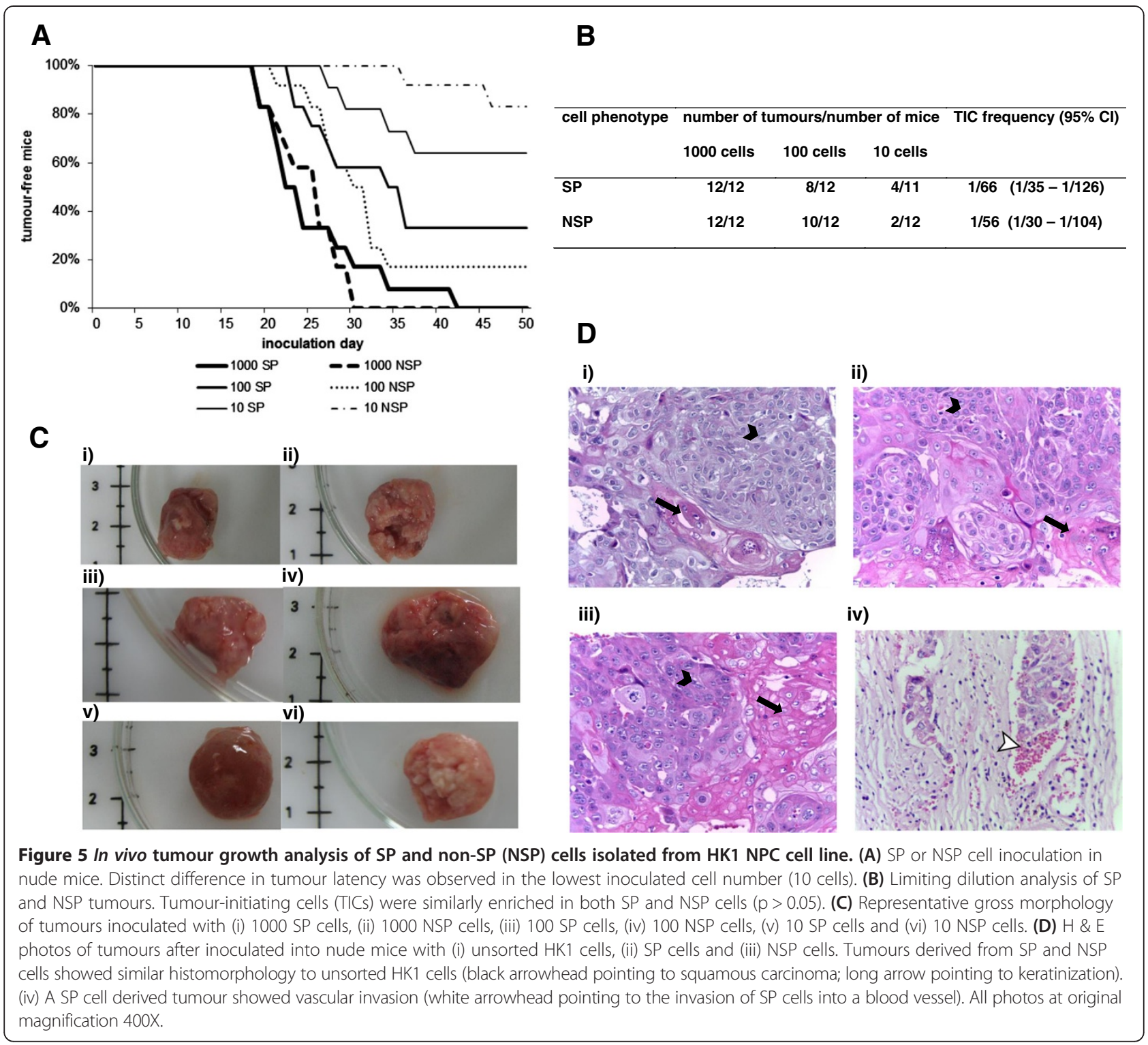

studied. Wang et al. utilized CNE-2 cells which had been reported to be contaminated with a partial genome of HeLa, whereas HK1 NPC cells used in this study was verified by STR profiling to be free from HeLa cell contamination [18]. Secondly, we used FTC as a dye efflux inhibitor to identify ABCG2-specific SP cells from HK1 and xeno-284 cells, instead of verapamil, a classic inhibitor for ABCB1-expressing cells $[15,16]$ which showed no inhibition in our study but was used in the above mentioned study [17]. Our negative finding was in line with Zhang et al. which reported that ABCG2 was not a CSC marker for NPC [25]. Lastly, the length of tumour assessment time in in vivo was different. Mice inoculated with CNE-2 SP and NSP cells were euthanized 4 weeks after inoculation, whereas our mice were observed for 7 weeks. As the incubation time was relatively short, there remains a possibility of CNE-2 NSP cells giving rise to tumour in mice after 4 weeks of inoculation.

\section{Conclusions}

Our study shows that ABCG2-specific SP cell subpopulation is found in HK1 NPC cells, a cell line generated from well-differentiated recurrent NPC but they are minimal in xeno-284 cells, a xenograft derived from poorly differentiated metastatic NPC. In spite of in vitro evidence proposing that CSCs from HK1 can be identified by the SP approach, in vivo validation study showed that both SP and NSP cells of HK1 had similar tumourigenicity potential. Therefore, we conclude that the SP approach alone cannot identify CSCs accurately in HK1 cells. 


\section{Materials and methods}

\section{Cell line and culture conditions}

HK1 NPC cells were cultured in fully-supplemented RPMI 1640 medium containing 10\% fetal calf serum and $1 \%$ penicillin/streptomycin (Gibco, NY, USA) in a $5 \% \mathrm{CO}_{2}$ incubator at $37^{\circ} \mathrm{C}$. The cells were confirmed to be mycoplasma-free by periodical testing with Venor GeM Mycoplasma Detection Kit for Conventional PCR (Minerva Biolabs, Berlin, Germany).

\section{Digestion of xenograft sample}

Xeno-284 NPC xenograft was freshly harvested and rinsed with cold sterile PBS supplemented with $1 \mathrm{X}$ antibiotic/ antimycotic (Gibco, NY, USA). After removing visible blood capillaries and/or necrotic tissue, the xenograft tissue was minced finely in the presence of $1 \mathrm{X}$ collagenase/ dispase solution (Roche, Mannheim, Germany). The mixture was incubated for $60 \mathrm{~min}$ in a $5 \% \mathrm{CO}_{2}$ incubator at $37^{\circ} \mathrm{C}$ with constant agitation. The cell suspension and undigested xenograft pieces were separated by sieving through $40 \mu \mathrm{m}$ cap strainer. After a brief centrifugation step, the cell pellet was resuspended in RBC lysis buffer (Qiagen, Hilden, Germany) and, the cells were rinsed with sterile PBS prior to cell count and viability check.

\section{Hoechst 33342 staining assay}

HK1 NPC cells at a logarithmic growth phase were detached by Accutase (Millipore, MA, USA) and washed at least once with PBS prior to Hoechst 33342-staining. A modification of Goodell's method was used to stain both HK1 and xeno-284 NPC cells [10]. Briefly, the cells were re-suspended at a concentration of $1 \times 10^{6}$ cells per $\mathrm{mL}$ in warm HBSS buffer (Gibco, NY, USA) supplemented with $2 \%$ fetal calf serum and 10mM HEPES ("HBSS+"). Hoechst 33342 dye (Molecular Probes, OR, USA) was added into the cell suspension at a final concentration of $5 \mu \mathrm{M}$ in the presence or absence of the ABCG2 inhibitor, fumitremorgin $\mathrm{C}$ (FTC) at a final concentration of 1 $\mu \mathrm{M}$ (Sigma, MO, USA) or verapamil (ABCB1 inhibitor) at a final concentration of 50 and $100 \mu \mathrm{M}$ (Sigma, MO, USA). The cells were incubated for $90 \mathrm{~min}$ in a $37^{\circ} \mathrm{C}$ water bath with intermittent mixing. Excessive Hoechst 33342 dye was removed from the cell suspension by washing with cold HBSS+. The resulting pellet was resuspended to a final concentration of $1-2 \times 10^{6}$ cells per $\mathrm{mL}$ with cold $\mathrm{HBSS}+$. In order to delineate host mouse cells, xeno-284 cells were further stained with $\mathrm{H} 2 \mathrm{Kd}-\mathrm{PE}$ antibody (1:10, BD Pharmingen, MA, USA) for $30 \mathrm{~min}$ at $4^{\circ} \mathrm{C}$. Propidium iodide (PI, $2 \mu \mathrm{M}, \mathrm{BD}$ Pharmingen, MA, USA) was added to stained HK1 NPC cells and, 7-AAD (BD Pharmingen, MA, USA) to Hoechst-stained xeno-284 cells for determination of cell viability. The stained cells were analyzed and sorted in a BD FACSAria II SORP cytometer (BD Biosciences, MA, USA) equipped with a
$355 \mathrm{~nm}$-UV laser power of $50 \mathrm{~mW}$. The Hoechst 33342 fluorescence was detected via a 405/20 band-pass filter (Hoechst Blue) and a 670 long pass filter (Hoechst Red). The sorted SP and NSP cells were collected in fullysupplemented RPMI medium. Presence of cells is reported as percentages of single, viable SP or NSP cells. Subsequent experiments were performed using HK1 NPC cells as xeno-284 NPC xenograft had too few SP cells for sorting.

\section{Clone morphology assay}

Sorted cells were plated at a low cell density of 50 cells per well in a 96-well culture plate containing fullysupplemented RPMI medium. Upon seeding, microscopic inspection of each well was performed to ensure there was no cell clumping. Once the cells were adherent, the culture medium was changed every two days. The numbers of holoclones, meroclones and paraclones were counted on day 8 post-sorting. Data were recorded from three independent experiments with 12 replicate wells per SP or NSP cells in each experiment.

\section{Aldehyde dehydrogenase (ALDH) assay}

Sorted cells were left for overnight recovery in the culture conditions as mentioned above. The cells were detached using Accutase and ALDH staining was performed with the ALDEFLUOR staining kit (Stem Cell Technologies, Vancouver, Canada). Briefly, $0.25 \times 10^{6}$ cells were resuspended in ALDEFLUOR assay buffer containing $1.5 \mu \mathrm{M}$ ALDH activated substrate, BAAA and incubated for 45 mins at $37^{\circ} \mathrm{C}$. Half of each sorted sample was treated with $15 \mu \mathrm{M}$ DEAB, an inhibitor of ALDH. The stained cells were washed, resuspended in cold ALDEFLUOR assay buffer containing $2 \mu \mathrm{M}$ PI and analyzed in BD FACSCalibur (BD Biosciences, MA, USA). Data were obtained from five independent experiments.

\section{Asymmetric division assay}

Ten thousand sorted cells were recultured for three weeks in fully-supplemented RPMI medium. Hoechst 33342 staining was performed as described above, and the cells were analyzed in a BD FACSAria II SORP cytometer. Data were recorded from three independent experiments.

\section{Proliferation assay}

Sorted cells were left to recover from the sorting process in a $5 \% \mathrm{CO}_{2}$ incubator at $37^{\circ} \mathrm{C}$ for approximately two hours. The "recovered" cells were seeded at 3000 cells per $200 \mu \mathrm{L}$ into each well of the E-plate 16 (Roche, Mannheim, Germany). Cell index values were recorded over a period of 125 hours with an interval of 1 hour for the first day, followed by every 6 hours for the remaining experiment by the xCELLigence System's Real time Cell Analyzer (RTCA) DP instrument (Roche, Mannheim, 
Germany). Cell index values represent measurements of electrical impedance of monitored cells which reflect cell growth (number and viability), morphology and adhesion ability. The cell index values of SP and NSP cells at each time point were then normalized to the control cells (HK1 cells which were only stained with PI and sorted from PI negative gate). Data were obtained from three independent experiments.

\section{Stem cell gene expression study}

Quantitative RT-PCR was performed using Human Stem Cell and Stem Cell Signalling PCR Arrays (SABiosciences, MD, USA). Total RNA was isolated using TRIzol (Invitrogen, CA, USA) and cleaned with RNeasy extraction kit (Qiagen, CA, USA). The quality and concentration of the extracted RNA was determined with NanoDrop 8000 spectrophotometer (Thermo Scientific, DE, USA) and only samples with a 260/280 ratio of $\sim 1.8$ were used for reverse transcription. Total RNA (100 ng) was reverse-transcribed using the accompanying First Strand Synthesis Kit and cDNA was amplified using SYBR green/ROX master mix in the ABI7500 Fast Real-Time thermal cycler (Applied Biosystems, CA, USA). Genes with Ct values of more than 35 were removed from analysis. Ct value of each gene was normalized against the geometric mean $\mathrm{Ct}$ value of five housekeeping genes $(\mathrm{Ct}$ geoHK $)$, with the formula $2^{-\mathrm{dCt}}$ $(\mathrm{dCt}=\mathrm{Ct}$ gene of interest $-\mathrm{Ct}$ geoHK $)$. The normalized $x$ values were used to calculate fold change ratios between SP and NSP. Data were obtained from three independent experiments.

\section{In vivo tumourigenecity assay}

Inoculations of SP and NSP cells into nude mice were performed in a limiting dilution manner: 1000, 100 and 10 cells. Sorted cells were mixed with BD Matrigel Basement Membrane Matrix (BD Biosciences, MA, USA) and inoculated subcutaneously into 5 - 6-week-old nude mice (Balb/c nu/nu). Tumour latency data, defined as the period between the inoculation day to the first day of tumour detection, were recorded. Once a palpable growth was detected, tumour volume was recorded every two days. Tumour volume $\left(\mathrm{mm}^{3}\right)$ was calculated from $0.5 \times$ (width $\times$ length ${ }^{2}$ ). All tumours were harvested once the length or width reached $10 \mathrm{~mm}$ or on day 50 post-inoculation. Limiting dilution analysis was performed using the Extreme Limiting Dilution Analysis (ELDA) software (http://bioinf.wehi. edu.au/software/elda/). Data for each group were from three independent experiments. All mice experiments were approved by the Animal Care and Use Committee of the Ministry of Health, Malaysia.

\section{Haematoxylin and eosin (H \& E) staining}

Four-micrometre formalin-fixed, paraffin-embedded tissue sections from SP and NSP tumours were mounted on plain glass slides and stained with $\mathrm{H}$ \& E. Histopathological observations were made under a light microscope by a pathologist and all tumours were confirmed to be NPC.

\section{Statistical analysis}

Data are reported as the mean \pm standard deviation (SD) or boxplot with whiskers showing the 5 - 95 percentile. All statistical analyses were performed using paired Student's $\mathrm{t}$ test from the GraphPad Prism 5 software (GraphPad Software Inc., CA, USA), except for clone morphology which used unpaired Student's t test. A p-value of $<0.05$ was deemed to be statistically significant.

\section{Additional files}

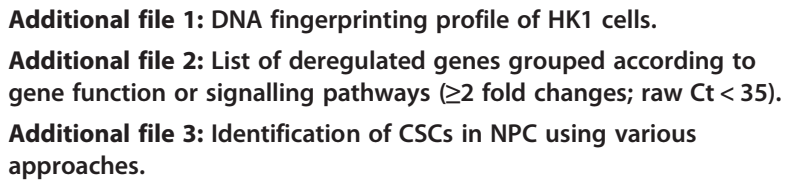

Competing interests

The authors declare that they have no competing interests.

\section{Authors' contributions}

SLLH, LPT, WCZ, MA and ASBK participated in the overall design of the study. SLLH optimized, planned and carried out most of the experiments with some assistance from JJ. SLLH, LPT, JJ and SCP analyzed the data. SLLH, LPT, SCP and ASBK interpreted the results. SLLH, LPT, CCN, MA and ASBK drafted the manuscript. All authors had read and approved the final manuscript.

\section{Acknowledgements}

We thank the Director- General of Health for his permission to publish this paper and the Director of the Institute for Medical Research for her support. We are also extremely grateful to Bing Lim (Genome Institute of Singapore, GIS) for his scientific insights and guidance, Lau Shin Hin (IMR) for her expert advice, Nurul Ashikin Mohamed Shahrehan, Nur Syazwani Mohd Sharif, Tai Lin Chu, Norazlin Abdul Aziz, Nurhanani Razali and Tiong Weng Ng (IMR) for their technical assistance. This project received funding from the Ministry of Health of Malaysia (JPP-IMR 09-037).

\section{Author details}

${ }^{1}$ Molecular Pathology Unit, Cancer Research Centre, Institute for Medical Research, Jalan Pahang, 50588 Kuala Lumpur, Malaysia. ${ }^{2}$ Institute of Biological Sciences, Faculty of Science, University of Malaya, 50603 Kuala Lumpur, Malaysia. ${ }^{3}$ Faculty of Medical Sciences, UCSI University, 1 Jalan Menara Gading, UCSI Heights, 56000 Cheras, Malaysia. ${ }^{4}$ Genome Institute of Singapore, 60 Biopolis Street, \#02-01, Genome 138672, Singapore. ${ }^{5}$ Current address: Faculty of Medicine, University of Malaya, 50603 Kuala Lumpur, Malaysia.

Received: 19 May 2014 Accepted: 22 September 2014

Published online: 09 October 2014

\section{References}

1. Lo KW, To KF, Huang DP: Focus on nasopharyngeal carcinoma. Cancer Cell 2004, 5(5):423-428.

2. Pua KC, Khoo AS, Yap YY, Subramaniam SK, Ong CA: Gopala Krishnan G, Shahid H, Malaysian Nasopharyngeal Carcinoma Study G: Nasopharyngeal Carcinoma Database. Med J Malaysia 2008, 63(Suppl C):59-62

3. Khoo ASB, Pua KC: Diagnosis and clinical evaluation of nasopharyngeal carcinoma. In Nasopharyngeal Carcinoma: Keys for Translational Medicine and Biology. Edited by Busson P. Austin: Landes Bioscience; 2013:1-9. 
4. Wei WI, Sham JS: Nasopharyngeal carcinoma. Lancet 2005, 365(9476):2041-2054.

5. Clarke MF, Dick JE, Dirks PB, Eaves CJ, Jamieson CH, Jones DL, Visvader J, Weissman IL, Wahl GM: Cancer stem cells-perspectives on current status and future directions: AACR Workshop on cancer stem cells. Cancer Res 2006, 66(19):9339-9344.

6. Alison MR, Lin WR, Lim SM, Nicholson LJ: Cancer stem cells: in the line of fire. Cancer Treat Rev 2012, 38(6):589-598.

7. Moore N, Lyle S: Quiescent, slow-cycling stem cell populations in cancer: a review of the evidence and discussion of significance. J Oncol 2011, 2011:396076.

8. Cao L, Zhou Y, Zhai B, Liao J, Xu W, Zhang R, Li J, Zhang Y, Chen L, Qian H, Wu M, Yin Z: Sphere-forming cell subpopulations with cancer stem cell properties in human hepatoma cell lines. BMC Gastroenterol 2011 11(1):71

9. Akunuru S, Palumbo J, Zhai QJ, Zheng Y: Rac1 targeting suppresses human non-small cell lung adenocarcinoma cancer stem cell activity. PLoS One 2011, 6(2):e16951

10. Goodell MA, Rosenzweig M, Kim H, Marks DF, De Maria M, Paradis G, Grupp SA, Sieff CA, Mulligan RC, Johnson RP: Dye efflux studies suggest that hematopoietic stem cells expressing low or undetectable levels of CD34 antigen exist in multiple species. Nat Med 1997, 3(12):1337-1345.

11. Kato K, Takao T, Kuboyama A, Tanaka Y, Ohgami T, Yamaguchi S, Adachi S, Yoneda T, Ueoka Y, Kato K, Hayashi S, Asanoma K, Wake N: Endometrial cancer side-population cells show prominent migration and have a potential to differentiate into the mesenchymal cell lineage. Am J Patho 2010, 176(1):381-392.

12. Fukaya R, Ohta S, Yamaguchi M, Fujii H, Kawakami Y, Kawase T, Toda M: Isolation of cancer stem-like cells from a side population of a human glioblastoma cell line, SK-MG-1. Cancer Lett 2010, 291(2):150-157.

13. Lim YC, Oh SY, Cha YY, Kim SH, Jin X, Kim H: Cancer stem cell traits in squamospheres derived from primary head and neck squamous cell carcinomas. Oral Oncol 2011, 47(2):83-91.

14. Wu C, Alman BA: Side population cells in human cancers. Cancer Lett 2008, 268(1):1-9.

15. Duan Z, Brakora KA, Seiden MV: Inhibition of ABCB1 (MDR1) and ABCB4 (MDR3) expression by small interfering RNA and reversal of paclitaxel resistance in human ovarian cancer cells. Mol Cancer Ther 2004, 3(7):833-838.

16. Robey RW, Shukla S, Steadman K, Obrzut T, Finley EM, Ambudkar SV, Bates SE: Inhibition of ABCG2-mediated transport by protein kinase inhibitors with a bisindolylmaleimide or indolocarbazole structure. Mol Cancer The 2007, 6(6):1877-1885.

17. Wang J, Guo LP, Chen LZ, Zeng YX, Lu SH: Identification of cancer stem cell-like side population cells in human nasopharyngeal carcinoma cell line. Cancer Res 2007, 67(8):3716-3724.

18. Chan SY, Choy KW, Tsao SW, Tao Q, Tang T, Chung GT, Lo KW: Authentication of nasopharyngeal carcinoma tumor lines. Int J Cancer 2008, 122(9):2169-2171.

19. Huang DP, Ho JH, Poon YF, Chew EC, Saw D, Lui M, Li CL, Mak LS, Lai SH, Lau WH: Establishment of a cell line (NPC/HK1) from a differentiated squamous carcinoma of the nasopharynx. Int J Cancer 1980, 26(2):127-132.

20. Alison MR, Guppy NJ, Lim SM, Nicholson LJ: Finding cancer stem cells: are aldehyde dehydrogenases fit for purpose? J Pathol 2010, 222(4):335-344.

21. Visvader JE, Lindeman GJ: Cancer stem cells: current status and evolving complexities. Cell Stem Cell 2012, 10(6):717-728.

22. Wu A, Luo W, Zhang Q, Yang Z, Zhang G, Li S, Yao K: Aldehyde dehydrogenase 1, a functional marker for identifying cancer stem cells in human nasopharyngeal carcinoma. Cancer Lett 2013, 330(2):181-189.

23. Lun SW, Cheung ST, Cheung PF, To KF, Woo JK, Choy KW, Chow C, Cheung CC, Chung GT, Cheng AS, Ko CW, Tsao SW, Busson P, Ng MH, Lo KW: CD44+ cancer stem-like cells in EBV-associated nasopharyngeal carcinoma. PLoS One 2012, 7(12):e52426

24. Zhuang HW, Mo TT, Hou WJ, Xiong GX, Zhu XL, Fu QL, Wen WP: Biological characteristics of CD133(+) cells in nasopharyngeal carcinoma. Oncol Rep 2013, 30(1):57-63.

25. Zhang H, Liu W, Feng $X$, Wang L, Jiang $X$, Liu D, Zhang L, Zhu B, Zhou W, Jia W, Li G, Ren C: Identification of ABCG2 (+) cells in nasopharyngeal carcinoma cells. Oncol Rep 2012, 27(4):1177-1187.
26. Locke M, Heywood M, Fawell S, Mackenzie IC: Retention of intrinsic stem cell hierarchies in carcinoma-derived cell lines. Cancer Res 2005, 65(19):8944-8950.

27. Li H, Chen X, Calhoun-Davis T, Claypool K, Tang DG: PC3 human prostate carcinoma cell holoclones contain self-renewing tumor-initiating cells. Cancer Res 2008, 68(6):1820-1825.

28. Zhou ZH, Ping YF, Yu SC, Yi L, Yao XH, Chen JH, Cui YH, Bian XW: A novel approach to the identification and enrichment of cancer stem cells from a cultured human glioma cell line. Cancer Lett 2009, 281(1):92-99.

29. Chen S, Xu Y, Chen Y, Li X, Mou W, Wang L, Liu Y, Reisfeld RA, Xiang R, LV $D$, Li N: SOX2 gene regulates the transcriptional network of oncogenes and affects tumorigenesis of human lung cancer cells. PLoS One 2012, 7(5):e36326.

30. Wu XQ, Huang C, He X, Tian YY, Zhou DX, He Y, Liu XH, Li J: Feedback regulation of telomerase reverse transcriptase: new insight into the evolving field of telomerase in cancer. Cell Signal 2013, 25(12):2462-2468.

31. Singh RR, Kunkalla K, Qu C, Schlette E, Neelapu SS, Samaniego F, Vega F: $A B C G 2$ is a direct transcriptional target of hedgehog signaling and involved in stroma-induced drug tolerance in diffuse large B-cell lymphoma. Oncogene 2011, 30(49):4874-4886.

32. Port RJ, Pinheiro-Maia S, Hu C, Arrand JR, Wei W, Young LS, Dawson CW: Epstein-Barr virus induction of the Hedgehog signalling pathway imposes a stem cell phenotype on human epithelial cells. J Pathol 2013, 231(3):367-377.

33. Dembinski $J$, Krauss $S$ : Characterization and functional analysis of a slow cycling stem cell-like subpopulation in pancreas adenocarcinoma. Clin Exp Metastasis 2009, 26(7):611-623.

34. Buczacki S, Davies RJ, Winton DJ: Stem cells, quiescence and rectal carcinoma: an unexplored relationship and potential therapeutic target. Br J Cancer 2011, 105(9):1253-1259.

35. Huang B, Huang YJ, Yao ZJ, Chen X, Guo SJ, Mao XP, Wang DH, Chen JX, Qiu SP: Cancer stem cell-like side population cells in clear cell renal cell carcinoma cell line 769P. PLoS One 2013, 8(7):e68293.

36. Mitsutake N, Iwao A, Nagai K, Namba H, Ohtsuru A, Saenko V, Yamashita S: Characterization of side population in thyroid cancer cell lines: cancer stem-like cells are enriched partly but not exclusively. Endocrinology 2007, 148(4):1797-1803.

37. Qi W, Zhao C, Zhao L, Liu N, Li X, Yu W, Wei L: Sorting and identification of side population cells in the human cervical cancer cell line HeLa. Cancer Cell Int 2014, 14(1):3

38. Peickert S, Waurig J, Dittfeld C, Dietrich A, Garbe Y, Kabus L, Baumann M, Grade M, Ried T, Kunz-Schughart LA: Rapid re-expression of CD133 protein in colorectal cancer cell lines in vitro and in vivo. Lab Invest 2012, 92(11):1607-1622.

doi:10.1186/s12935-014-0101-0

Cite this article as: Hoe et al:: Evaluation of stem-like side population cells in a recurrent nasopharyngeal carcinoma cell line. Cancer Cell International 2014 14:101.

\section{Submit your next manuscript to BioMed Central and take full advantage of:}

- Convenient online submission

- Thorough peer review

- No space constraints or color figure charges

- Immediate publication on acceptance

- Inclusion in PubMed, CAS, Scopus and Google Scholar

- Research which is freely available for redistribution 